Virginia Junqueira 1

Umberto Catarino Pessoto 1

Jorge Kayano 1,2

Paulo Roberto Nascimento 1

I racema Ester do Nascimento Castro 1,3

Jucilene Leite da Rocha 1

Marcelo Fernando Terence 1

Roberta Cristina Boaretto 1

Lauro Cesar I banhes 1

Carlos Tato Cortizo 1

Luiza Sterman Heimann 1

\section{Equidad en la salud: evaluación de políticas públicas en Belo Horizonte, Minas Gerais, Brasil, 1993-1997}

\author{
Equity in the health sector: evaluation of public \\ policy in Belo Horizonte, M inas Gerais State, \\ Brazil, 1993-1997
}

1 Núcleo de Investigação em Si stemas de Serviços de Saúde, Instituto de Saúde, Secretaria de Estado da Saúde de São Paulo. Rua Santo Antonio 590, São Paulo, SP 01314-000, Brasil. nisis@isaude.sp.gov.br 2 Instituto de Estudos, Formação e Assessoria em Políticas Sociais. Rua Cônego Eugênio Leite 433, São Paulo, SP 05414-010, Brasil.

3 Distrito de Saúde Cidade Líder, Secretaria Municipal de Saúde de São Paulo. Rua General Jardim 36 São Paulo, SP 01223-010, Brasil.
Abstract This article evaluates government measures to reduce inequity in the health sector in Bel o Horizonte from 1993 to 1997. Our hypothesi s is that a municipal administration committed to equity can reduce disparities in heal th with the support of the Unified National Heal th System (SUS). The methodology used an urban quality of life index in Belo Horizonte to detect social inequalities in living conditions, as well as differences between the component indices in the infant mortal ity rate. Other municipal measures were assessed according to the investment resulting from the implementation of a participatory local budget and open planning process. The urban quality of life index appeared to be an appropriate measure for orienting municipal administration. The infant mortality rate proved to be a good indicator for measuring inequality in health. There was a reduction in IMR and mortal ity reducing gaps in the districts studied. We observed greater investment of physical and financial resources in the districts with the lowest urban quality of life index, and it can thus be stated that the municipal administration reduced the prevailing inequalities.

Key words Equity; Living Conditions; Health Policy; Evaluation of Public Health Interventions

Resumen El artículo evalúa el resultado de las acciones del gobierno en la reducción de las desi gualdades en el ámbito sanitario en Belo Horizonte de 1993 a 1997. La hipótesi s es que un gobierno municipal comprometido con la equidad puedereducir desi gualdades, apoyándose en el Sistema Único de Salud. Trabajamos con el Índice de Calidad deVida Urbana (IQVU), usado en el municipio para reconocer las desigualdades en las condiciones de vida y el diferencial de los coeficientes de mortalidad infantil (CMI), neonatal e pos-neonatal en los nueve distritos sanitarios. Otras acciones fueron medidas por la ejecución de obras municipales, por medio del Presupuesto Parti ci pativo y la Programación Abierta. EI IQVU se mostró apropiado para orientar la acción del gestor municipal. El CMI se mostró un buen indicador para medir la desi gualdad en salud. Hubo reducción del CMI y de las brechas de mortali dad en los distritos estudiados. Verificamos mayor inversión de recursos físi cos y financieros en Ios distritos de IQVU más bajo, pudiéndose afirmar que en el estudiado, el gobierno municipal redujo desigualdades.

Palabras clave Equidad; Condiciones deVida; Política de Salud; Evaluación de Acciones de Salud Pública 


\section{Introducción}

La lucha por la redemocratización en Brasil en los años 80, y su otro lado de la moneda en el sector de la Salud, Ilega a su punto culminante con la realización de la 8a Conferencia Nacional de Salud, en 1986, en búsqueda de la intervención en las resoluciones de la Asamblea Nacional Constituyente, apareciendo sobre el papel un capítulo exclusivo de Salud en la Constitución de 1988, cuando se instituye el Sistema Único de Salud (SUS). Sus principios - la universalidad, la integridad de la atención y la equidad, así como sus presupuestos organizativos, - la descentralización de la gestión, con dirección única en cada esfera de gobierno, a través de la municipalización; la regionalización, la jerarquización, la participación de la comunidad, por medio de fórums paritarios y deliberativos (Consejos Municipales, Estatales y Nacional de Salud) y el carácter complementario del sector privado, fueron reglamentados por la Ley 8.080/90 (Brasil, 1990a) y la Ley 8.142/ 90 (Brasil, 1990b), que crearon instrumentos y estructuras que dieron a los estados $y$ municipios una mayor capacidad gerencial de sus sistemas de salud.

No obstante, en la década de los 90 , en la mira de la estabilización económica y ajuste fiscal, el gobierno brasileño promovió cortes consecutivos en el presupuesto federal, alcanzando el área de salud. El presupuesto aprobado para el Ministerio de Salud en 1997 fue de R\$20,4 billones, de los cuales sólo R \$17,59 bilIones fueron liberados (G. C. Carvalho, 1998, comunicación personal); En 1998 el presupuesto ejecutado fue de 19,3 billones, no ultrapasando el ejecutado en 1995, que fue de 19,4 billones (G. C. Carvalho, 1998, comunicación personal).

Las dificultades derivadas de la política económica, el gran número de municipios del país, la enorme diversidad regional, los conflictos entre los diversos intereses existentes en el área sectorial, constituyen obstáculos técnicos y políticos para el avance de la implementación del SUS. Incluso así, se observan resultados positivos en los municipios que consiguieron cualificarse en los niveles más avanzados de gestión descentralizada, - la semiplena con aumento de la capacidad gerencial y de la oferta de acciones y servicios; aproximación a las necesidades de salud de la población, y un nuevo padrón de relación entre los sectores público y privado y la mayor interacción y participación de la comunidad en el control público del sistema de salud (Heimann et al., 1998). De esta forma, partimos de la hipótesis de que, incluso en un ambiente político-económico adverso, la acción del gobierno municipal comprometido con la equidad puede reducir las desigualdades en el sistema de salud local.

\section{O bjetivos generales}

Evaluar el resultado de las acciones de gobierno dirigidas a la reducción de desigualdades en salud en el municipio de Belo Horizonte en el período de 1993 a 1997.

\section{Objetivos específicos}

Identificar las desigualdades sociales y de salud en las diferentes regiones de la ciudad; Identificar las acciones de gobierno y de salud que indujeron a la variación en la distribución de la mortalidad infantil; medir y analizar la variación de la mortalidad infantil en las diferentes regiones de la ciudad.

\section{Cuadro teórico}

La equidad en salud es entendida en este trabajo como la superación de desigualdades que son evitables y consideradas injustas, implicando que las necesidades diferenciadas de la población sean atendidas por medio de acciones gubernamentales también diferenciadas (Whitehead, 1990). Subyace a este concepto el entendimiento moderno de que las desigualdades entre las personas no se producen "naturalmente", sino que se crean por el proceso histórico y por el modo de producción y organización de las sociedades.

En la literatura, el reconocimiento de las desigualdades en salud se hace evidente, entre otros, por estudios de mortalidad (Behm, 1980; Carvalho \& Costa, 1998; Castellanos, 1994; Charlton, 1987; Charlton et al., 1983; Monteiro, 1986; Monteiro \& Nazário, 1995; Silva et al., 1999).

Analizando los perfiles de mortalidad de los países de América Latina desde 1970, Castellanos (1994:3) se apunta la influencia de las "desi gual dades sociales, del cambio en la estructura de edad de la población, del proceso deurbanización, de los cambios en la composición de la fuerza de trabajo y en el mercado de trabajo, de los cambi os en los nivel es educacional es de la población y de los cambios en la organización de los servicios de salud". Afirma que las medias decrecientes de las tasas de mortalidad específicas en las franjas más jóvenes y el au- 
mento de la expectativa de vida esconden "una realidad de profunda desigual dad entre paísesy entre sectores sociales en el interior de cada país" (Castellanos, 1994:2). El autor propone que las tasas de mortalidad de cada país o grupo sean comparadas no sólo con aquellas obtenidas anteriormente en el mismo país, sino con las obtenidas en países o grupos de países que comparten circunstancias semejantes. Las diferencias entre las tasas calculadas para el país o grupo observado y aquellas que son tomadas como referencia son denominadas Brechas Reducibles de Mortalidad.

Castellanos (1991) cita también los trabajos de Anderson (1990, apud Castellanos, 1991), en América Central y México, sobre la vigilancia de la mortalidad infantil y otros problemas de salud, donde una población con alta mortalidad infantil fue seleccionada como centinela para vigilar el impacto de acciones dirigidas a mejorar la supervivencia de los niños. No sólo el reconocimiento de las desigualdades es posible, sino también diferentes enfoques analíticos de los estudios de mortalidad son realizados para acompañar y evaluar la reducción de las desigualdades en el ámbito de la salud. Aparte de esto, en sociedades que cuentan con sistemas fiables de estadísticas vitales, como los Estados Unidos y la mayoría de los países europeos, es común el uso de series históricas de mortalidad infantil de distintas clases sociales para supervisar la evolución de la equidad social. En Brasil, varios estudios han demostrado la existencia de diferenciales de mortalidad infantil entre estratos sociales (Victora et al., 1988), regiones del país o incluso en áreas de una misma ciudad (Monteiro, 1986; Paim et al., 1987).

\section{Metodología}

La especificidad del objeto llevó a la opción metodológica del estudio del caso.

Los criterios de selección que orientaron la elección del municipio - en el caso de que fueran: de gran porte; relevancia nacional; mayor capacidad gerencial - gestión semiplena hace por lo menos cuatro años -; gran complejidad asistencial en la red de servicios; existencia de informaciones evidenciando desigualdades intra-urbanas en las condiciones de vida de la población; gobierno municipal orientado para la equidad y continuidad administrativa. De entre las 26 capitales brasileñas, Belo Horizonte y Porto Alegre reunían la mayoría de los criterios. Seleccionamos Belo Horizonte por el tiempo mayor en gestión semiplena en el SUS y por la razón de poseer un sistema de información construido para el reconocimiento de las desigualdades intra-urbanas (Índice de Calidad de Vida Urbana - IQVU/Belo Horizonte).

El año base para la investigación fue 1993 por la razón de que era: (a) el inicio de la administración municipal; (b) el año propuesto por la Secretaría Municipal de Salud para cualificarse a la gestión semiplena; (c) año en el que el gobierno municipal ya disponía de informaciones para el reconocimiento de desigualdades intra-urbanas. El diseño dela investigación fue de observación anterior y posterior a una intervención y trabajamos con el período de 1993 a 1997.

En 1994 el municipio fue cualificado y asumió la modalidad de gestión semiplena del sistema de salud. Pasó, entonces, a la condición de gestor del SUS de una red compuesta por 58 hospitales, totalizando 7.835 camas; 121 centros de salud; nueve policlínicas; un puesto de primeros auxilios municipal; tres unidades de urgencia; cuatro unidades de referencia especializada; dos centros de referencia de salud mental, además de una extensa red de apoyo diagnóstico.

EI IQVU/Belo Horizonte fue construido a partir de un conjunto de 11 variables, treinta componentes y 75 indicadores en los áreas de vivienda, infraestructura, salud, educación, servicios urbanos, seguridad, abastecimiento, medioambiente, cultura, deporte y asistencia social. Los indicadores están localizados geográficamente en 81 unidades espaciales (Unidades de Planeamiento - UP), a partir del Sistema de Informaciones Geográficas del Procesamiento de Datos de Belo Horizonte - PRODABEL - (Prefeitura Municipal de Belo Horizonte, 1996).

Utilizamos el IQVU/Belo Horizonte para el reconocimiento de las desigualdades en las condiciones de vida a través de la variación de los coeficientes de: mortalidad infantil (CMI), mortalidad neonatal (CMNN) y mortalidad posneonatal (CMPNN) en las diferentes regiones de la ciudad, como expresión de las desigualdades en salud, cuyas variaciones fueron analizadas posteriormente a través de las Brechas Reducibles de Mortalidad (Castellanos, 1994).

En el caso del CMI, la serie histórica de Belo Horizonte presentaba el declive gradual desde 1982 pasando de 54,8/ 1.000 nacidos vivos para 39,2/ 1.000 en 1992. En cambio, cuando es analizada en el ámbito de los Distritos Sanitarios (DS), que forman un número total de nueve en la ciudad, se evidenciaban desigualdades acentuadas entre las diversas regiones del municipio. 
Clasificamos los DS en grupos de condiciones de vida semejantes, a través de la división en "quartis" de las UP (81), lo que nos permitió distinguir tres grupos de Distritos. El primer grupo, donde predominaban UP con IQVU más elevados, concentraba el mayor número de servicios, así como la mayor renta media de la población de la ciudad, y estaba formado sólo por el Distrito de Salud Centro Sur. Pertenecían al segundo grupo, de UP con IQVU medios, Ios Distritos de Salud de Pampulha, Noroeste, Oeste, Este y Nordeste. El tercer grupo, de UP con IQVU más bajos, estaba formado por los Distritos de Salud de Barreiro, Venda Nova y Norte. Aparte del hecho de no tener condiciones operacionales para trabajar con los nueve DS, Ia tipología de los distritos descrita encima fue un recurso metodológico considerado fundamental para el delineamiento de la investigación y el reconocimiento de las acciones que intervendrían en el binomio desigualdad/ equidad, expresado en la mortalidad infantil de las diferentes áreas y grupos sociales de Belo Horizonte.

El grupo de distritos con mejores condiciones de vida fue excluido del estudio por tener sólo un Distrito, imposibilitando la puesta en operatividad del concepto de brecha reducible. En el grupo de Distritos de condiciones de vida media, con cinco distritos, fue necesaria la elección de dos de ellos. En este grupo, el Distrito Nordeste, fue el único que presentó para los tres coeficientes de mortalidad (la infantil, neonatal y pos-neonatal), en el año 1997, un comportamiento fuera de lo esperado. De esta forma, se definió que este distrito sería un Distrito-Caso, a ser comparado, en el mismo grupo, al Distrito de Pampulha, seleccionado por presentar en el estudio de la mortalidad infantil la mayor tasa de reducción del coeficiente en el período de 1993 a 1997. En el grupo de distritos de peores condiciones de vida, dos fueron seleccionados: Barreiro, a semejanza de Pampulha, por presentar la mayor tasa de reducción del CMI en el período; y Venda Nova, por su comportamiento negativo fuera de lo esperado, para el CMI y CMNN en 1995, y en 1993 para el CMPNN.

Para el análisis de la variación en la caída de la mortalidad, utilizamos el estudio de la significación de las diferencias entre los CMI de los Distritos, a través de la Razón de Mortalidad Estandarizada - Standartized Mortality Ratio (SMR). Los Distritos que no acompañaron la variación de la mortalidad dentro de los padrones esperados serían aquellos que potencialmente necesitaban un estudio en profundidad para la explicación de las diferencias, lo que sucedió con los Distritos de Barreiro, Norte, Nordeste y Venda Nova.
Seleccionados los Distritos-Casos fueron definidos los procesos, variables e indicadores para el estudio aproximativo tanto de las acciones de gobierno intersectoriales como las del propio sector de salud.

El estudio de las acciones de gobierno fue realizado a través de los instrumentos del aprovechamiento de recursos utilizado por el gobierno municipal: el Presupuesto Participativo (OP), que contiene el plan de inversiones en obras, así como los planes sectoriales según las deliberaciones de las Asambleas Regionales (Prefeitura de Belo Horizonte, 1998) y la Programación Abierta (PA), que reúne los recursos aprovechados conforme la orientación exclusiva del gobierno.

Las variables e indicadores utilizados para medir las acciones intersectoriales de gobierno estuvieron relacionadas con: (a) toma de decisión, medida por el porcentaje de obras de infraestructura urbana definidas por el proceso de decisión participativo (OP) y por la PA que fueron realizados, (b) aprovechami ento de recursos, medida por el montante de recursos aprovechados según las prioridades definidas por el proceso de decisión; (c) distribución de recursos, medida por el gasto per capita hecho por el gestor, según el origen presupuestario (PA o OP).

Las obras fueron agrupadas en dos tipos: (1) las obras intersectoriales: servicios de canalización de arroyos, drenaje, contención de laderas y pavimentación después del drenaje y canalización; y (2) las obras de salud: ampliación, reforma o construcción, sea de policlínicas, centros de salud, puestos de socorro u hospitales.

El estudio de las acciones en el sector salud fue realizado según dos dimensiones: las acciones asi stencial es y las acciones gerencial es del sistema local de salud. En el primer caso fueron analizadas: la oferta de servicios relacionada al planeamiento familiar, prenatal, asistencia al parto y al recién nacido y el sistema de referencia entre servicios de atención básica y hospitalaria (referencia y contrarreferencia entre servicios prenatales y red hospitalaria). Fueron también analizados indicadores de resultado de las acciones asistencial es como: las tasas de recién nacido de bajo peso, de prematuros, de parturientas adolescentes, tipo de parto y Apgar de cinco minutos. En la evaluación del proceso gerencial fueron seleccionadas variables e indicadores relativos a recursos humanos (variación numérica, perfil de los trabajadores y entrenamientos realizados para la capacitación de personal) y la participación y control social. En este artículo, procuramos ex- 
clusivamente indicar sus implicaciones directas en las acciones de salud.

El estudio de las brechas reductibles tuvo como objetivo cuantificar el resultado de las acciones de gobierno, tanto intersectoriales como las del sector salud, para la reducción de las desigualdades sociales expresadas en las variaciones de los indicadores de salud. Para el cálculo de los márgenes escogemos el menor coeficiente obtenido por un distrito en todo el período. Este procedimiento fue aplicado tanto al CMI, como a sus componentes - CMPNN y CMNN. Así los valores-padrón definidos para el cálculo de los márgenes fueron: CMI - 17,94, presentado por el Distrito de Salud de PampuIha en 1997; CMPNN - 3,94, presentado por el Distrito de Salud de Pampulha en 1997; CMNN - 13,02, presentado por el Distrito de Salud de Pampulha en 1993. De esta forma, el cálculo de los márgenes de mortalidad infantil y sus componentes fueron hechos a partir de las siguientes ecuaciones:

$$
\text { Margen } \mathrm{CMI}=\frac{\begin{array}{c}
\text { (CMI de un determinado } \\
\text { DS) }-17,94
\end{array}}{17,94}
$$

(CMPNN de un determinado

$$
\text { Margen CMPNN }=\frac{\text { DS) }-3,94}{3,94}
$$

(CMNN de un determinado

$$
\text { Margen } \mathrm{CMNN}=\frac{\mathrm{DS})-13,02}{13,02}
$$

Las fuentes de datos secundarios fueron: Sistema de Información de Mortalidad (SIM), Sistema de Información de Nacidos Vivos (SI NASC), Sistema de Información Socio-demográfico, Sistema de Información de la ciudad de Belo Horizonte, Sistema de Información de Salud del municipio, Sistema Nacional de Informaciones Hospitalarias y Ambulatorias (SIA, SIH/SUS). Utilizamos también como fuentes: programa de gobierno, actas y documentos de los foros participativos, programas, proyectos, etc. Realizamos todavía entrevistas semi-estructuradas con Directores de Distritos y representantes de los usuarios en los Consejos de Distritos de Salud.

En cuanto al plan deanálisis, se articuló el comportamiento del coeficiente de la mortalidad infantil con las acciones de salud y las acciones de gobierno. Para la reducción de las brechas en la mortalidad infantil esperábamos encontrar variación positiva de las acciones de gobierno y de salud; en el caso de acentuación de las brechas esperábamos variación negativa das acciones de gobierno y de salud; por fin, en la alternativa de no alteración de los márgenes, las acciones de gobierno y de salud podrían variar contradictoriamente o mostrar variación negativa concomitante.

\section{Resultados}

En cuanto a las cuestiones metodológicas, inicialmente construimos el IQVU de los DS a partir del IQVU/Belo Horizonte por UP - Ia unidad mínima de desagregación de los datos de mortalidad infantil. Así, fue recalculado el IQVU/Belo Horizonte por UP expurgándose los componentes referentes a la variable salud, a saber, la tasa de supervivencia con hasta un año de edad y el peso al nacer igual o superior a $2.500 \mathrm{~g}$. Como las UP diferían en el tamaño poblacional, calculamos también su peso proporcional en relación a la población total del respectivo distrito. Esta ponderación originó el IQVU - Relativo a cada UP. EI IQVU - del Distrito fue obtenido a partir de la suma de los valores del IQVU-Relativo a las UP de cada DS.

No obstante, siendo el IQVU una medida de calidad de vida del lugar, y no necesariamente de las personas que lo habitan, podría incurrirse en una falsa aproximación de la realidad al tomarse este índice como medida de desigualdad de condiciones de vida de la población. Para que superásemos esta dificultad, utilizamos el Índice de Vulnerabilidad Social de Belo Horizonte (IVS), núcleo central del Mapa dela Exclusión Social de Belo Horizonte con informaciones que analizan atributos de individuos, no del lugar (Nahas et al., 1997, 1999; Nahas \& Martins, 1995).

EI Test de Correlación de Pearson entre el IQVU/Belo Horizonte (banco original), el IQVU - Recalculado (calculado por nosotros, sin los indicadores de mortalidad infantil y peso del recién nacido) y el IVS para cada UP demostró fuerte correlación y alta significación entre los índices cotejados, confirmando, así, que áreas con bajos IQVU abrigan en su interior poblaciones depauperadas y víctimas de las desigualdades sociales, de condiciones de vida, engendradas por las relaciones de producción. Esta fuerte correlación nos permite afirmar, con seguridad, que el IQVU - del Distrito es un buen instrumento de descripción de las desigualdades sociales en nuestro municipio-caso.

Presentaremos ahora los principales resultados en cada una de las dimensiones estudiadas: 
Las acciones de gobierno

La evaluación de la acción de gobierno fue hecha a través del análisis de las obras realizadas, recursos financieros aprobados por el OP y definidos por la PA, entre 1994 y 1997 (Tabla 1).

Conforme la Tabla 1, si consideramos solamente las obras originadas del OP, verificamos que el $30,8 \%$ y el $29,5 \%$ - los mayores porcentajes - de los recursos fueron empleados en Venda Nova y Nordeste; mientras que el $22,4 \%$ y el $17,2 \%$, en Barreiro y Pampulha, respectivamente. La lógica del gestor expresa en la distribución de recursos vía PA se muestra diversa de la lógica participativa del OP. Barreiro consume el $52,2 \%$ de los recursos de PA; $26,2 \%$ son consumidos en el Nordeste; el 19,9\% en Venda Nova y sólo el 1,6\% en Pampulha. O sea, en la práctica del OP los recursos se distribuyen de manera más igualitaria, sin embargo, en la del PA más de la mitad de los recursos fueron destinados a una única regional (Barreiro), exactamente una de las que tienen peores condiciones de vida, indicando posiblemente orientación más equitativa.

Con relación a la distribución de los recursos según el gasto 'per capita' hecho por el gestor (Tabla 2), en el período, verificamos que la inversión por habitante se reveló mayor en el Distrito de Salud de Barreiro, seguido de Venda Nova, Nordeste y Pampulha, en este orden, exactamente el mismo orden establecido por el IQVU en los Distritos-Casos, tomados de forma decreciente. O sea, a mayor IQVU, menor el gasto 'per cápita' de los recursos totales.

En el gasto 'per capita' de los recursos, considerándose los recursos destinados por el OP en cada Distrito, verificamos un dispendio igual en Venda Nova y Pampulha, mayores que en el

Tabla 1

Recursos ejecutados en acciones de gobierno por origen presupuestario.

Cuatro distritos de salud seleccionados. Belo Horizonte, 1994 a 1997.

\begin{tabular}{|c|c|c|c|c|}
\hline \multirow{2}{*}{$\begin{array}{l}\text { Distritos } \\
\text { de Salud }\end{array}$} & \multicolumn{2}{|c|}{ Presupuesto participativo } & \multicolumn{2}{|c|}{ Programación abierta } \\
\hline & Valor en $\mathrm{R} \$$ & $\%$ & Valor en $\mathrm{R} \$$ & $\%$ \\
\hline Pampulha & $2.524 .737,10$ & 17,2 & $242.159,82$ & 1,6 \\
\hline Nordeste & $4.328 .498,65$ & 29,5 & $3.914 .679,27$ & 26,2 \\
\hline Barreiro & $3.285 .322,55$ & 22,4 & $7.797 .379,70$ & 52,2 \\
\hline Venda Nova & $4.520 .735,75$ & 30,8 & $2.978 .201,26$ & 19,9 \\
\hline $\begin{array}{l}\text { Belo Horizonte } \\
\text { (Total) }\end{array}$ & $14.659 .294,05$ & 100,0 & $14.932 .420,05$ & 100,0 \\
\hline
\end{tabular}

Fuente: Superintendencia para el Desarrollo de la Capital,

Prefectura Municipal de Belo Horizonte.
Nordeste y en Barreiro. Ya aquellos originados en Ia PA, Barreiro, Nordeste y Venda Nova ostentan los mayores índices de gasto per capita, siendo el de Barreiro el doble del gasto de los demás, y Pampulha surge con el menor de los gastos por habitante. Esta ordenación es semejante a la del IQVU, de forma inversa (menor IQVU, mayor gasto 'per capita'), y también semejante a la ordenación de los distritos como a la distribución de los recursos totales oriundos del PA.

En síntesis, en la búsqueda de tendencias del comportamiento del administrador, como al tratamiento dispensado a los distritos de Belo Horizonte, encontramos los siguientes resultados: (1) mayor aporte total (OP + PA) de recursos por distrito en aquellos tres de menor calidad de vida; (2) mayor destinación de recursos oriundos de OP a los mismos tres distritos; (3) mayor destinación de recursos oriundos del PA para los tres distritos de menor IQVU; (4) mayor gasto 'per capita' en los tres distritos de menor IQVU; (5) dar prioridad flagrante de recursos del PA al distrito de menor calidad de vida; y (6) proporcionar prioridad relativa para los recursos del PA para el distrito de segundo menor IQVU. Sin embargo, el destino mayor de recursos de PA para Barreiro y el relativo otorgamiento de privilegios en relación con los recursos de PA para Venda Nova tal vez se hayan producido por las características de las obras necesarias.

\section{Las acciones en el sector salud}

En 1993, la administración municipal, a partir de un diagnóstico situacional, definió como prioridad la atención al niño, trazando políticas públicas que atendiesen a sus necesidades. Había una clara intención de los gestores del SUS en reducir las desigual dades sociales y de salud a partir de la reorganización de la asistencia a la gestante y a los niños con prioridad para las áreas de riesgo definidas en el proceso de la división por distritos (Campos et al., 1998).

Este proceso de reorganización de la atención, denominado Proyecto Vida, fue implantado a partir de 1994 y contemplaba, además del cambio del proceso de trabajo, acciones dirigidas a la captación precoz de la clientela y acompañamiento de las gestantes en el período prenatal; encauzamiento de la asistencia al parto para la maternidad según las referencias por distrito; visitas a las maternidades y/ o visitas domiciliares para la captación de los niños con criterios de riesgo para el acompañamiento en los Centros de Salud; asistencia integral a 
Población, gastos 'per capita' y gastos 'per capita' según el origen presupuestario, en los Distritos-Casos. Belo Horizonte, 1994 a 1997.

\begin{tabular}{lccccc}
\hline Distritos & $\begin{array}{c}\text { Población en } \\
\text { mil habitantes }\end{array}$ & $\begin{array}{c}\text { Gastos totales } \\
\text { en mil R\$ }\end{array}$ & $\begin{array}{c}\text { Gastos 'Per } \\
\text { Capita' (R\$) }\end{array}$ & $\begin{array}{c}\text { 'Per Capita' } \\
\text { OP (R\$) }\end{array}$ & $\begin{array}{c}\text { 'Per Capita' } \\
\text { PA (R\$) }\end{array}$ \\
\hline Pampulha & 120 & 2.767 & 23 & 21 & 2 \\
Nordeste & 251 & 8.243 & 33 & 17 & 16 \\
Barreiro & 237 & 11.082 & 47 & 14 & 33 \\
Venda Nova & 218 & 7.499 & 34 & 21 & 14 \\
\hline
\end{tabular}

Fuente: Superintendencia para el Desarrollo de la Capital, Prefectura Municipal de Belo Horizonte.

la salud del niño, programa de prevención y combate a la desnutrición; entre otras.

El Sub-Proyecto de Vigilancia a la Mortalidad Infantil implantó mecanismos de acompañamiento especialmente para los recién-nacidos de madres residentes en áreas de riesgo. Fueron agregados en la definición del recién nacido (RN) bajo vigilancia de algunos criterios obtenidos a partir del banco de datos del SINASC: RN por debajo de $2.500 \mathrm{~g}$ (bajo peso), analfabetismo de la madre y madre adolescente, aspectos ya identificados en la literatura como favorecedores de un mayor riesgo de óbito neo-natal.

\section{Evaluación de las acciones asistenciales}

En relación al planeamiento familiar, de los indicadores propuestos, solamente conseguimos obtener informaciones, desagregadas por distrito, sobre la incidencia del embarazo en la infancia y en la adolescencia. Identificamos una clara tendencia de elevación en la participación de esta franja de edad el total de partos realizados por el SUS, compatible con el aumento observado en $\mathrm{BH}$ y en el país como un todo, cuya tasa está en torno al 20\%. En la media de los cuatro Distritos, parturientas niñas y adolescentes pasan del $14,8 \%$ en 1993 al $17,7 \%$ en 1997. No obstante, la frecuencia de gestantes con menos de 14 años, franja de mayor riesgo biológico, y también de gestaciones indeseadas, siguió una tendencia a la baja. Se destacan los Distritos de Pampulha, que redujo en $83,3 \%$ la incidencia de partos en la franja de edad de 10 a 14 años y Venda Nova, en 53,3\%, contra una media de los cuatro Distritos del $50 \%$.

El incentivo al acompañamiento de la gestante en la red dio prioridad a la captación precoz pues este factor es decisivo en la calidad de la situación prenatal. Hubo un aumento signi- ficativo del número de gestantes acompañadas de julio de 1996 a abril de 1997 (de 4.384 a 8.179), en todos los DS. La media de gestantes que iniciaban la etapa prenatal en el primer trimestre de gestación estaba en torno al 35\% del total de gestantes acompañadas en los tres Distritos donde pudimos obtener los datos, Pampulha, Nordeste y Barreiro. Sin embargo, este esfuerzo en la ampliación del acompañamiento prenatal en la red pública municipal tal vez no se revele significativo frente al número de gestantes del municipio, cuya estimación para 1996 era de cerca de 50 mil.

Esta insuficiencia en la cobertura se refleja en los resultados encontrados en los cuatro distritos, ya que en cuanto a los RN de bajo peso (por debajo de 2.500g), en los cuatro distritos, el porcentaje se mantuvo en torno al $10 \%$ de 1993 a 1997, lo que supera los valores observados en Brasil como un todo, que era del orden del 7,5\% en 1997 (SINASC). La baja eficacia de los DS estudiados en reducir la incidencia de bajo peso al nacer, dando prioridad a los grupos de riesgo como las gestantes con menos de 14 años, se expresa también en la mantenimiento de altas tasas de adolescentes, que aumentó en todos los DS; con una media del $4 \%$, en 1993, para posicionarse en torno al $6 \%$ en 1997, val or mayor que al general del país, que en 1997 fue del 5\% (SINASC).

En relación a la asistencia al parto, la tasa de cesáreas aumentó en todos los distritos entre 1993 y 1997. Este aumento fue diferenciado en los cuatro distritos estudiados, siendo que Pampulha fue el que presentó mayor aumento entre 1993 y 1997 (13,7 puntos porcentuales), seguido de Barreiro (3,8\%), Venda Nova $(3,24 \%)$ y por fin Nordeste con 2,83\% más en el período. Este aumento se dio a costas de la reducción en el número de partos con fórceps, principalmente en el Distrito de Salud Pampulha, y en la reducción de partos normales en Ven- 
da Nova (2,69\%), Barreiro (2,64\%) y Nordeste (1,62\%). El Distrito de Pampulha aumentó en 2,94 puntos porcentuales la tasa de partos normales. En los hospitales privados contratados por el SUS en los distritos-casos esta tasa varió de $27,3 \%$ a 41,4\% en 1998. En el mismo año, el porcentaje de cesáreas de los hospitales privados no contratados del SUS varió mucho más, llegando a alcanzar el 92,3\% en uno de estos hospitales.

Otro indicador seleccionado para que evaluemos la calidad de las acciones de prenatal y asistencia al parto fue el Apgar, los primeros 5 minutos de vida. Para este análisis utilizamos la siguiente clasificación: Apgar entre 8 y 10 normal; entre 5 y 7 - regular; y Apgar de 4 o menos - bajo.

Los datos muestran una tendencia de reducción de los nacimientos con Apgar regular (en los 4 Distritos, del 3,4\% en 93 al 2,3\% en 97) y también de los nacidos vivos con Apgar debajo del 4 (de 1,0\% en 1993 al 0,7\% en 1997). Al analizar la variación del porcentaje de reciénnacidos con Apgar regular y bajo, entre 1997 y 1993, verificamos que el distrito que presentó la mayor reducción del Apgar regular fue Venda Nova (3\%) seguido de Pampulha (1,6\%), Nordeste $(1,0 \%)$ y Barreiro $(0,9 \%)$. En lo que se refiere al Apgar bajo, la mayor reducción fue en Barreiro $(0,7 \%)$, seguido de Venda Nova $(0,5 \%)$ y Pampulha (0,2\%). El Distrito de Nordeste tuvo un aumento del $0,2 \%$ de recién nacidos con Apgar bajo en el período estudiado.

En lo que toca a la expansión de la oferta de servicios para estas acciones de salud, verificamos que el número de Unidades Básicas de Salud (UBS) y otros servicios que realizaban planeamiento familiar, como está previsto en las acciones del Proyecto Vida, aumentó en el período de 1993 a 1997 en todos los distritos-casos, destacando especialmente Venda Nova. Respecto a los servicios de prenatal hubo un aumento de la oferta en los DS Nordeste y Barreiro y la reducción de un servicio en Pampulha y Venda Nova debido a que fue desmantelado. Observamos todavía un aumento significativo en la atención prenatal de riesgo, que en el 93 no existía en ninguno de los distritos estudiados.

En cuanto a los lechos obstétricos, localizadas y que están disponibles para el área de alcance de los Distritos, se verificó la reducción importante en Pampulha (80 en 1993 y cero en 1997), como resultado de que una unidad de maternidad fue desmantelada; no hubo alteración en el Nordeste y Barreiro; y en Venda Nova hubo un aumento de más que el doble de lechos para partos, debido a la ampliación de los hospitales del área.
Un factor importante de organización de la oferta de los lechos fue la implantación, en 1994, de la Central de Internamiento para asistencia al parto. Esta forma permitió mayor accesibilidad a las parturientas a los lechos de maternidad, principalmente las de aquellas moradoras en áreas desprovistas de este tipo de servicio, así como una mejor organización del sistema de referencia y contrarreferencia entre UBS y hospitales.

En cuanto a los lechos de la Unidad de Terapia Intensiva (UTI) neonatal, en 1993, sólo existían en el DS Nordeste y, en 1997, estas camas ya estaban siendo puestas a disposición de todos los distritos-casos por la Central de Internación, conforme datos de las entrevistas. Las unidades de atención a urgencias fueron ampliadas en Venda Nova y servicios de apoyo -farmacia, laboratorios en los diferentes distritos y central de material esterilizado- se expandieron en el período, principalmente en el Nordeste, Venda Nova y Barreiro.

La secretaria también amplió las camas para recién-nacidos de riesgo de 70 , en 1994, a 117 en 1996. Además de esta inversión, el Consejo Municipal de Salud aprobó la complementación de la tabla de pagos del SUS para aquellas maternidades que tuviesen sus dependencias equipadas para la asistencia al recién-nacido de riesgo, de acuerdo con criterios establecidos por la Comisión Perinatal, de la Secretaría Municipal de Salud.

El análisis de la oferta de los servicios presentado encima revela que la acción del gobierno realizada en el sector salud fue mayor en los Distritos de Nordeste, Barreiro y Venda Nova, siendo que estos dos últimos componen el grupo de distritos de peores condiciones de vida y el DS Nordeste está en el grupo de condiciones de vida media. Este aumento diferenciado de la oferta local, privilegiando las regiones de la ciudad con peores condiciones de vida sugiere una tendencia redistributiva de la oferta en la implementación del SUS en el nivel local en el período estudiado.

En lo que se refiere a la atención al niño, fueron implementadas acciones para el acompañamiento del crecimiento y desarrollo de niños menores de cinco años, con el Programa de Prevención y Combate a la Desnutrición. Las actividades se iniciaron en agosto/93, en colaboración con la Secretaria de Abastecimiento y, desde 1995, con la Pastoral de la Tierra. El programa se inició con 51 UBS y, en 1997, estaba implantado en 126 unidades.

A partir de 1996 con la elaboración de un protocolo de acompañamiento de desnutridos, asociando la entrada del niño en el proyecto al 
grado nutricional en el transcurso del cuatrimestre, se posibilitó mayor rigor en la sistematización de las informaciones sobre asistencia al niño. De este sistema de evaluación derivó en un nuevo registro de los desnutridos en la red de UBS. En julio de 1996, se incluyeron a todos los niños en el protocolo de acompañamiento. Este nuevo registro significó un aumento absoluto de niños inscritos: de 6.485 en julio de 1996, a 18.092 niños en abril de 1997.

Entre los niños desnutridos evaluados en 1995 por el Programa, se observó que el 39\% presentaron cambios del grado de desnutrición para mejor, el 56\% se mantuvieron en el mismo grado de desnutrición, además con una mejora clínica acentuada, o sea, disminución de problemas de piel, reducción de episodios de Infección Respiratoria Aguda (IRA) y de diarrea y reducción del número de internamientos hospitalarios; un $5 \%$ se presentaron peor que el cuadro inicial.

En relación al impacto del Programa sobre la mortalidad infantil, se nota que el combate a la desnutrición, como causa básica, contribuyó para la reducción de la mortalidad. Hubo una reducción significativa en el número absoluto y relativo de los óbitos por desnutrición, responsable del 12,3\% (78) de los óbitos en 1993, al 6,5\% (29) de los óbitos en 1995. Según el informe de evaluación del Proyecto Vida, estos resultados reflejan el impacto de un conjunto de acciones desarrolladas en el ámbito de la atención materno-infantil. Llama la atención también la reducción de los óbitos por infecciones intestinales, así como las pneumonías y broncopneumonías (BCP). Por tanto, podemos afirmar que la reducción de la mortalidad infantil fue una prioridad de hecho asumida por las administraciones y los Consejos de Salud de los distritos estudiados. A pesar de los gestores de distritos y del Consejo Municipal de Salud, según las actas, no participaron de la formulación del Proyecto Vida, su implementación reveló una fuerte adhesión.

El análisis de las brechas reductibles de la mortalidad infantil

La serie histórica del CMI de Belo Horizonte por distritos sanitarios en el período de 1993 a 1997, mostró una tendencia de caída de la mortalidad infantil en el municipio. Esta reducción se dio de forma heterogénea entre los distritos, siendo que el DS de Barreiro fue el que presentó la mayor reducción en el período (Tabla 3).

El CMPNN, a su vez, en el Municipio de Belo Horizonte como un todo decrece continua-
Tabla 3

Brechas reductibles del coeficiente de mortalidad infantil de los Distritos

Sanitarios. Belo Horizonte, 1993 a 1997.

\begin{tabular}{lccccc}
\hline \multirow{2}{*}{ Distrito } & \multicolumn{5}{c}{ Brechas reductibles } \\
& 1993 & 1994 & 1995 & 1996 & 1997 \\
\hline Pampulha & 0,50 & 0,61 & 0,79 & 0,32 & 0 \\
Nordeste & 0,80 & 0,74 & 0,53 & 0,22 & 0,64 \\
Barreiro & 1,25 & 0,78 & 0,73 & 0,15 & 0,06 \\
Venda nova & 0,72 & 0,90 & 0,29 & 0,65 & 0,09 \\
\hline
\end{tabular}

Fuente: Secretaría Municipal de Salud de Belo Horizonte y Núcleo de Investigación en Sistemas de Servicios de Salud.

mente en el período, así como en la mayoría de los distritos, destacando también al DS Barreiro, que presentaba en 1993 el mayor coeficiente de CMPNN y mostró la mayor reducción, del orden del 75\%. Ya el componente neonatal (CMNN) se elevó en 1994, lo que explica el aumento de la mortalidad infantil del municipio de Belo Horizonte en este año. En Ios años siguientes, en cambio, se produjo una reducción continua, al mismo tiempo, en ritmo mucho menor del que la reducción del componente CMPNN, al punto de que la reducción entre los años de 1993 y 1997 se colocó por debajo de la mitad de la reducción alcanzada por la mortalidad infantil. Mientras que, el análisis del CMNN en los DS no se muestra ningún padrón regular indicativo de esta tendencia.

EI DS Pampulha presentaba en 1993 el menor margen, pero se evidencia una elevación de este valor en los dos años siguientes, de modo que en 1995 tenía el mayor margen entre todos los DS de Belo Horizonte. En 1996 este margen fue bastante reducido, hasta hacerse nulo en 1997 (Tabla 4). Los demás distritos presentan también una evolución irregular del margen, con reducciones seguidas de elevaciones en el período. El Nordeste, después de tres años seguidos de una reducción del margen, presentó una expresiva elevación en 1997, siendo el DS con el mayor margen. Venda Nova, a su vez, presentó resultados todavía más irregulares, pues aumentó el margen en 1994, lo redujo en 1995, presentó una nueva elevación en 1996, seguida de una reducción en 1997, cuando tenía la segunda menor brecha entre todos en este año.

El análisis comparativo del comportamiento de las brechas entre los cuatro distritos evidencia una diferencia del orden de 0,53 entre Ios distritos de menor IQVU - Barreiro y Venda Nova - en 1993, el cual se redujo a 0,03 en 1997, a favorable a Barreiro. La caída indica que la 
Brechas reductibles de la mortalidad infantil neonatal y pos neonatal de los Distritos Sanitarios del Municipio de Belo Horizonte - 1993 a 1997.

\begin{tabular}{|c|c|c|c|c|c|c|c|c|c|c|}
\hline \multirow[t]{3}{*}{ Distritos } & \multicolumn{10}{|c|}{ Brechas reductibles } \\
\hline & \multicolumn{2}{|c|}{1993} & \multicolumn{2}{|c|}{1994} & \multicolumn{2}{|c|}{1995} & \multicolumn{2}{|c|}{1996} & \multicolumn{2}{|c|}{1997} \\
\hline & CMNN & CMPNN & CMNN & CMPNN & CMNN & CMPNN & CMNN & CMPNN & CMNN & CMPNN \\
\hline Pampulha & 0 & 2,51 & 0,25 & 2,20 & 0,62 & 1,80 & 0,43 & 0,27 & 0,08 & 0 \\
\hline Nordeste & 0,37 & 2,67 & 0,42 & 2,23 & 0,39 & 1,37 & 0,06 & 1,03 & 0,52 & 1,28 \\
\hline Barreiro & 0,53 & 4,18 & 0,42 & 2,40 & 0,52 & 1,83 & 0,08 & 0,67 & 0,04 & 0,31 \\
\hline Venda Nova & 0,47 & 1,98 & 0,39 & 3,04 & 0,10 & 1,25 & 0,57 & 1,32 & 0,08 & 0,39 \\
\hline
\end{tabular}

Fuente: Secretaría Municipal de Salud de Belo Horizonte y Núcleo de Investigación en Sistemas de Servicios de Salud. CMNN = Coeficiente de Mortalidad Neonatal; CMPN N = Coeficiente de Mortalidad Pos-Neonatal

Tabla 5

Media de las brechas reductibles de los coeficientes de mortalidad infantil (CMI), neonatal (CMNN) y pos neonatal (CMPNN). Belo Horizonte, 1993-1997.

\begin{tabular}{llll}
\hline \multirow{2}{*}{ Año } & \multicolumn{3}{c}{ Media de las brechas reductibles } \\
& CMI & CM N & CMPN N \\
\hline 1993 & 0,84 & 0,36 & 2,87 \\
1994 & 0,81 & 0,41 & 2,60 \\
1995 & 0,61 & 0,41 & 1,70 \\
1996 & 0,42 & 0,31 & 1,11 \\
1997 & 0,28 & 0,22 & 0,67 \\
\hline
\end{tabular}

Fuente: Secretaría Municipal de Salud de Belo Horizonte y N úcleo de Investigación en Sistemas de Servicios de Salud, Instituto de Salud, Secretaría de Estado de la Salud de São Paulo.

desigualdad en salud entre los dos Distritos prácticamente desapareció al final del período. Lo mismo no sucedió en los Distritos de IQVU medio, Pampulha y Nordeste, cuya diferencia de 0,30 en 1993, pasó a 0,64, en 1997. Por otro lado, cuando comparamos la reducción de los márgenes de Barreiro y Venda Nova en relación con Pampulha, identificamos que los primeros (de menor IQVU) se aproximaron bastante a Pampulha (de mayor IQVU).

Para un mejor análisis del comportamiento de los coeficientes de mortalidad infantil, posneonatal y neonatal y, consecuentemente de las brechas entre los Distritos, en el conjunto del Municipio de Belo Horizonte, utilizamos el artificio de las medias de las brechas. La evolución de las brechas a lo largo de los cinco años sugiere una reducción de las desigualdades en salud entre los DS. Ella puede ser observada, tanto por la reducción del tamaño del mayor margen a lo largo de dos años, como por la significativa reducción de la media de las brechas presentados por los nueve Distritos en el período estudiado. Asimismo, los tres distritos con los menores márgenes en relación con el DS Pampulha, en 1997, son exactamente los tres distritos con los peores indicadores de condiciones de vida.

La reducción de las desigualdades en salud expresadas por la mortalidad infantil y observadas en el conjunto de los nueve Distritos, tuvo lugar también en los cuatro distritos-casos estudiados. En el caso de la mortalidad posneonatal el comportamiento de la media de los márgenes así como del tamaño del mayor margen es más expresivo incluso. Es bueno resaltar que este componente es sensible a las interferencias de variables que están fuera del control del sector salud. En este caso, de un nivel de 4,18 de margen, presentado por el Distrito de Barreiro, es de una media de 2,87, en 1993, se pasa a una media de 0,67 y para un margen de 1,39, en 1997, presentada por el DS Centro Sur. Nótese que el DS Centro Sur es el Distrito de mayor IQVU en el municipio.

En síntesis, sea en el análisis del comportamiento de las brechas por año sea por la media de las brechas, los datos demuestran tendencia de reducción de las diferencias entre los DS.

\section{Discusión}

Este artículo analizó el resultado de las acciones del gobierno municipal de Belo Horizonte en la reducción de las desigualdades en salud, en el período 1993 a 1997. La variación del coeficiente de mortalidad infantil, en las diferentes regiones de la ciudad, fue utilizada como proxy de resultados en términos de equidad, teniéndose en consideración las desigualdades sociales y de salud preexistentes, en las diferentes regiones de la ciudad, y las acciones in- 
tersectoriales y en la salud que pueden haber contribuido a este cambio.

Los principales resultados de este estudio apuntan que es posible - y necesario - utilizar la información disponible en el nivel local y construir indicadores que sean útiles tanto para identificar las desigualdades sociales y de salud existentes, como para supervisar los resultados de las acciones de gobierno (sectoriales e intersectoriales) para la disminución de las desigualdades entre distintos territorios de un mismo municipio.

Siendo así, una política de gobierno comprometida con la superación de las desigualdades en el nivel local es crucial para el alcance de las directrices del SUS - y la actuación del gestor es esencial para garantizar el equilibrio entre las demandas manifestadas por la población, los recursos disponibles y las necesidades identificadas política y técnicamente.

En el enfrentamiento entre el volumen de recursos y el número de obras realizadas, por Distrito, clasificados según la calidad de vida medida por el IQVU, se nota que el mayor voIumen de recursos financieros empleado tuvo lugar justamente en el distrito que presentó menor calidad de vida (menor IQVU) y que lo inverso se dio con el distrito con la más elevada calidad de vida.

La mitad de los recursos gastados en obras provinieron de la programación del OP. Este mecanismo fue responsable por la indicación de más de la mitad de las obras realizadas, en general, de porte pequeño, a juzgar por el coste medio, en el conjunto de los cuatro Distritos. En los tres distritos de menor IQVU, el coste medio de las obras presupuestadas en OP fue inferior a los demás, mientras que en PampuIha fue el triple. EI OP representó, por tanto, un mecanismo de distribución de recursos que reflejó las necesidades sentidas por la población, en cantidad y costes correspondientes a las necesidades presumidas, en las regiones de alta y de baja calidad de vida.

Aparentemente, teniendo la mayoría de sus necesidades básicas satisfecha, Pampulha acabó por demandar en los OPs obras de costo relativo muy superior al de los demás distritos y en cantidad muy inferior. Por otro lado, la distribución de los recursos totales presupuestados en OP se reveló relativamente equilibrada entre los cuatro distritos, y, siendo así, el OP, en el caso estudiado, se reveló un mecanismo de distribución igualitaria de los recursos, pero no necesariamente equitativa, pues no privilegió las regiones más pobres.

A través de la PA, el administrador hace llegar a la población la otra mitad de los recursos involucrados. Y, su decisión pendió a favor de Barreiro, uno de los distritos con peores condiciones de vida, destinando apenas el 1,6\% de los recursos al distrito de mayor IQVU (PampuIha). El coste medio de las obras originadas en PA demuestra una clara concentración prioritaria de recursos en los distritos de Barreiro y Venda Nova, los de menor IQVU, mientras que el coste medio de las obras de PA presupuestadas para el Nordeste y Pampulha fueron mucho inferiores. De esta forma, aparentemente el destino mayor de recursos al DS Barreiro, en términos absolutos, y al DSVenda Nova, en términos relativos, se dio por la necesidad de obras de gran porte en aquellas regionales, que no fueron contempladas en el OP. Estos datos apuntan la importancia del papel del gestor en la redistribución final de los recursos.

Otra tendencia en la distribución de recursos se refiere al tipo de obra realizada. Las obras de salud tuvieron un costo medio equivalente a menos de la mitad del coste de las obras intersectoriales. Reformas, ampliaciones y construcción de centros de salud representan costos seguramente inferiores a los de canalización de arroyos, drenaje, contención de laderas, pavimentación y, lo que es muy común, la realización de dos o tres de estos servicios en la misma obra. A juzgar por el número de obras y recursos empleados, aparentemente los Distritos estaban mucho más necesitados de obras intersectoriales: Barreiro realizó una obra de salud para cada 4,4 intersectoriales y esa proporción fue de 1:6 en el Nordeste, 1:29 en Venda Nova y 1:2 en Pampulha. Exceptuándose esta última regional, en que es reducido el número total de obras (sólo seis), la preocupación con, o la necesidad de, realización de obras en salud fue mayor en Barreiro, seguido del Nordeste. Acordémonos todavía de la mayor agilidad con que las obras de salud fueron implementadas en Barreiro que, en su mayoría, fueron presupuestadas en $\mathrm{OP}$, sugiriendo una clara preocupación comunitaria con la cuestión. En el Nordeste, también se dio una relativa prioridad por el OP a las obras de salud, pero tanto estas como las intersectoriales se hicieron efectivas tardíamente. En Venda Nova, poca cosa fue hecha en términos de obras de salud, donde el gestor destinó un cuidado específico al Distrito, pero limitado a la realización de obras intersectoriales. Fueron realizadas pocas obras de salud en Pampulha, y aunque ellas hayan sido presupuestadas por el administrador, tuvieron un costo medio de 1/ 5 del costo medio de las intersectoriales.

Existió el montaje de una red de servicios de salud en el nivel local, con preocupación en 
relación a la complejidad tecnológica necesaria para enfrentar el problema de la mortalidad infantil, en sus distintos niveles, evidenciando también aquí la tendencia redistributiva del gestor: los Distritos de peor calidad de vida fueron aquellos en que hubo una mayor expansión de la oferta. Por otro lado, al gunos servicios, como por ejemplo las farmacias de los Distritos, fueron implantadas en todos los Distritos.

Los indicadores analizados en relación a la asistencia prenatal evidencian que: (1) no se observaron diferencias importantes entre los Distritos-casos; (2) no se observaron variaciones importantes en el período de estudio; y (3) las variaciones no presentaron una regularidad que permitiese identificar la tendencia, ni para mejor, ni para peor. Sin embargo, la frecuencia de gestantes con menos de 14 años disminuyó en todos Ios DSs, lo que puede indicar mayor efectividad en las acciones de atención básica a la infancia y adolescencia.

En relación con la atención al parto, la Central de Internamientos implantada parece ser un avance, pues al ampliar y facilitar el acceso a camas para la población usuaria del SUS, favoreció a los habitantes de distritos que no disponen de camas hospitalarias suficientes en su territorio. Asimismo, este mecanismo generó un banco de datos que informa de las actividades de auditoría y control realizada por el nivel central de la Secretaría Municipal de Salud de Belo Horizonte (SMS-BH) y por los Distritos, que tuvo como resultado el desmantelamiento de algunos servicios. En compensación, la tasa de cesáreas aumentó en todos los DSs, pero el porcentaje de Apgar bajo o regular disminuyó revelando una mejoría en la asistencia al parto y nacimiento.

Los datos evidencian que las acciones de salud realizadas para la reducción de la Mortalidad Infantil en su componente neonatal, determinadas principalmente por las acciones gerenciales y por la asistencia al embarazo y al parto, fueron diferenciados en Ios Distritos, destacando Venda Nova, Barreiro Pampulha. El Distrito del Nordeste tuvo un desempeño desfavorable, en la medida en que la proporción de recién-nacidos que necesitaban cuidados intensivos aumentó. Estos datos confirman el análisis de tendencia de la serie histórica del CMNN.

Había, por tanto, una clara intención de los gestores del SUS en el Municipio de Belo Horizonte de reducir las desigualdades sociales y de salud en este grupo prioritario. Mientras tanto, la rede física de servicios de salud es necesaria pero no suficiente para impactar de forma po- sitiva en los indicadores de salud. De una manera general, los datos evidenciaron que, a pesar del proceso asistencial pueda haber contribuido para la caída de la mortalidad infantil, aunque es necesario mejorar su calidad, lo que debería incidir más fuertemente en el componente neonatal, incluyendo la atención al parto. Evidenciaron también que la práctica de evaluación de resultados del sistema de salud puede y debe ser implementada y que la discusión del perfil de mortalidad de los Distritos, y su variación, puede ser un indicador valioso para los gestores.

En lo que toca al análisis de las brechas reductibles del CMI, la técnica de aproximación utilizada permitió tanto un análisis del municipio como la comparación entre los Distritos. En el primer caso, identificamos que los márgenes en 1993 - sea del CMI o de cualquiera de sus componentes - se redujeron en el período estudiado, de forma continua y constante. Este hecho se expresa en la caída de las medias de las brechas de $1993(0,84)$ a $1997(0,28)$, significando que las desigualdades intra-urbanas fueron atenuadas. En el segundo caso, cuando comparamos los distritos pertenecientes al mismo grupo con el municipio, el comportamiento en el grupo de IQVU bajo se asemeja al ocurrido en el conjunto del municipio, o sea disminuyen mucho, volviéndose casi inexpresiva, en cual quiera de los indicadores estudiados. Por tanto, la desigualdad de los indicadores entre distritos de condiciones de vida semejantes prácticamente desapareció. Cuando analizamos el grupo de IQVU medio, verificamos que las brechas entre los Distritos de Pampulha y Nordeste presentan una elevación, especialmente en el grupo de IQVU bajo y en el municipio como un todo. Se resalta la gran aproximación que existe, en este período, entre los coeficientes de los dos distritos pertenecientes al grupo de IQVU bajo y el del Distrito de Pampulha. Cuando se analiza el comportamiento de las respectivas direcciones en los procesos participativos del OP y también la preocupación gerencial con la cuestión de la mortalidad infantil, estos tres distritos se distinguieron del Distrito del Nordeste, que fue el único en el que hubo un aumento del CMI y del CMNN por encima de los valores de los dos años anteriores. Esto ocurrió en 1997, cuando aquellos coeficientes subieron a 29,38 y 19,84 por mil nacidos vivos en comparación a los 27,39 e 18,0 de 1995, respectivamente. Mientras tanto, cuando analizamos el CMPNN del DS Nordeste, verificamos que su evolución, a pesar del aumento observado en 1997, mantiene la tendencia de caída. Esto permite inferir que la mayor irre- 
gularidad presentada por este Distrito se debe más al componente Neonatal, indicador sensible a las acciones del sistema y servicios de salud.

Caben aquí algunos comentarios sobre las dificultades metodológicas enfrentadas en el transcurso de la investigación. Podemos abordarlas en dos niveles, en el plano teórico y en el plano instrumental sea cual sea: recogida de datos, disponibilidad y análisis de datos cualitativos y cuantitativos. En el primer aspecto, se resalta la complejidad del objeto, de la cual derivan las limitaciones relativas a la necesidad de recurrir a recortes de la realidad estudiada. En el segundo aspecto, nos fijamos en las fragilidades y lagunas de las fuentes de datos secundarios y con las imprecisiones y subjetividades inherentes a la recogida de datos primarios. Tales óbices no deben impedir la realización de investigaciones, inclusive las de Servicios y Sistemas de Salud, en países como el nuestro siempre que sean adecuadamente identificados.

\section{Conclusión}

La propuesta de este trabajo no fue hacer una evaluación del sistema de salud en Belo Horizonte, sino identificar de qué manera acciones intersectoriales y sectoriales contribuyeron a la reducción de las desigualdades en salud en el municipio.

El estudio demostró que la información disponible en el nivel local puede ser utilizada para la identificación de desigualdades entre áreas distintas de un mismo municipio. Mostró todavía que existen formas de organizar los datos de la mortalidad infantil y sus componentes que permiten construir indicadores a ser utilizados como instrumentos para la supervisión tanto de las condiciones de vida y salud de la población, como de la efectividad delas acciones de gobierno realizadas. En síntesis, el análisis de los márgenes del CMI permite una aproximación preliminar sobre las desigualdades existentes y sugiere también las intervenciones requeridas, constituyéndose como una forma indirecta de mediación de los resultados de las acciones de gobierno, tanto las intersectoriales como las específicas del sector salud.

Observamos coherencia entre el comportamiento de la caída de la mortalidad infantil y de los márgenes en las áreas donde el gobierno municipal invirtió más, principalmente a través de la PA. Esto remite a la importancia del gestor en la administración local, una vez que los mecanismos participativos de decisión y control social son indicativos de las ansias y necesidades de la población, pero no pueden ser tomados de forma absoluta. La compatibilidad entre distintas demandas y el necesario equilibrio entre la disponibilidad financiera y las prioridades, sean sectoriales o no, requiere una visión más amplia de la situación global del municipio, que muchas veces se pierde en la focalización en un área o tipo de demanda específica.

En esta perspectiva, la principal contribución de este estudio está en evidenciar que la implementación de las directrices y principios del SUS, tales como universalidad, equidad, atención integral y participación social, adquiere en el nivel local gran significado, pues la reducción de las desigualdades sociales y de las desigualdades en salud dependen necesariamente de un proyecto de gobierno local comprometido con estas directrices. La implementación de programas específicos, dirigidos al acompañamiento y control de riesgos y agravios, reconocidos como problemas de salud en sus determinantes socio-económicos, culturales, ambientales y biológicos, en individuos y grupos sociales concretos, debe ser tomada como una forma de operatividad para la superación de esas desigualdades.

EI Proyecto Vida cumple con estos presupuestos en su formulación. Mientras que, para el acompañamiento y evaluación de sus resultados es necesario que se construya un subsistema de informaciones donde sea posible discriminar y cuantificar los grupos prioritarios en áreas específicas, refinando el análisis y proporcionando indicadores para la gestión local, que permitan la revisión y reorientación permanente de las acciones.

Algunas recomendaciones a los gestores derivan de este estudio y son sintetizadas a continuación.

- En relación a las desigualdades en salud: identificar a los grupos poblacionales que están en situación de desigualdad social en el proceso de conocimiento del territorio de las áreas de extensión de las unidades básicas de salud (configuración territorial).

- En relación con el sistema de información: (1) incorporar indicadores generales de desigualdad, como es el caso del IQVU, en el sistema de información del SUS municipal; (2) cuantificar el numerador y el denominador de los grupos identificados como prioritarios desde el punto de vista de la desigualdad social posibilitando el cál culo de indicadores de cobertura; (3) discriminar los datos relativos a los atendidos por la red del SUSy aquellos referentes a la clientela privada, de seguros y planos 
de salud; (4) incorporar en la rutina del sistema de información de la SMS-BH, en el nivel del distrito, los datos relativos al cuadro de personal, hoja de pago, gastos de costeo y de inversión, que son disponibles gracias a la Secretaría Municipal de Planeamiento;

- En relación con el planeamiento local (sectorial eintersectorial): (1) discutir la importancia de indicadores como la mortalidad infantil como referencia para la identificación de las desigualdades intra-urbanas; (2) priorizar acciones dirigidas para los grupos sociales considerados en situación de riesgo; (3) incorporar a lo cotidiano del trabajo el uso de indicadores que identifiquen las desigualdades sociales y en salud en el proceso decisorio, como el IQVU/ 1994 y el IQVU/ 1996; Ios datos del Mapa de la Exclusión Social; la mortalidad infantil y otros indicadores, subsidiando las discusiones de los Consejos Municipal, de Distritos y Locales de Salud y en el OP; (4) utilizar la variación de estos indicadores para evaluar el impacto de las acciones ejecutadas; (5) consolidar la rutina de totalización de las inversiones por Región Administrativa/ Distrito Sanitario, facilitando el acompañamiento y evaluación de las acciones de gobierno (tipos de obras, costos, tipo de presupuesto, etc.).

\section{Agradecimientos}

Este proyecto fue desarrollado por el Núcleo de Investigação em Sistemas de Serviços de Saúde, do Instituto de Saúde, Secretaria de Estado da Saúde de São Paulo, como parte del Programa Equity-oriented Health Policy Analysis in Latin America, implementado por la Red de Investigación en Sistemas y Servicios de Salud en el Cono Sur de América Latina, financiado por el Internacional Development Research Center, de Canadá. Agradecemos el soporte técnico brindado por Claudia Travassos, investigadora del Centro de Informação Científica e Tecnológica para a Saúde, Fundação Oswaldo Cruz y Celia Almeida, coordinadora del Programa y Secretaria Ejecutiva de la Red.

\section{Referencias}

BRASIL, 1990a. Ley 8.080, del 19 de septiembre de 1990. Versa sobre las condiciones para a promoção, proteção e recuperação da saúde, a organização e o funcionamento dos serviços correspondentes e dá outras providências. Brasília: Diário Oficial da República Federativa do Brasil, no 182, 20 set. pp. 18055-18059.

BRASIL, 1990b. Lei 8.142, de 28 de dezembro de 1990. Dispõe sobre a participação da comunidade na gestão do Sistema Único de Saúde - SUS e sobre as transferências intergovernamentais de recursos financei ros na área da saúde e dá outras providências. Brasília: Diário Oficial da República Federativa do Brasil, no 249, 31 dez. pp. 2569425695.

BEHM, H., 1980. Determinantes económicas y sociales de la mortalidad en América Latina. Revista Cubana deAdministración deSalud, 6:1-30.

CAMPOS, C. R.; MALTA, D. C.; REIS, A. T.; SANTOS, A. F. \& MERHY, E. E. (org.), 1998. Sistema Ú nico de Saúde em Belo Horizonte - Reescrevendo o Público. São Paulo: Xamã.

CARVALHO, B. G. \& COSTA, M. C. N., 1998. Mortalidade infantil e seus componentes em SalvadorBA, 1980-1991. Informe Epidemiológico do SUS, 4:35-41. 
CASTELLANOS, P. L., 1991. Proyecto: Sistemas Nacionales de Vigilancia de la Situación de Salud según Condiciones deVida y del Impacto delas Acciones de Salud y Bienestar. Washington, DC: Organización Panamericana de la Salud/Organizacion Mundial de la Salud. (mimeo.)

CASTELLANOS, P. L., 1994. Perfiles de Mortalidad, Nivel de Desarrollo e Inequidades Sociales en la Región de las Américas. Washington, DC: Organización Panamericana de la Salud/ Organización Mundial de la Salud. (mimeo.)

CHARLTON, J. R. H., 1987. Avoidable deaths and diseases as monitor of health promotion. In: Measurement in Health Promotion and Protection ( $T$. Abelin, Z. J. Brzezinski \& V. D. L. Carstairs, ed.), pp. 467-479, Copenhagen: World Health Organization/International Epidemiological Association.

CHARLTON, J. R. H.; SILVER, R.; HARTLEY, R. M. \& HOLLAND, W. W., 1983. Geographical variation in mortality from conditions amenable to medical interventions in England and Wales. Lancet, 3: 691-696.

CHAUÍ, M., 1980. A questão democrática. In: A Questão da Democracia (A. Wolfe, T. Santos, P. G. Casnova, H. J. Souza, E. Laclau \& M. S. Chauí, org.), Coleção Centro de Estudos de Cultura Contemporânea, pp. 139-172, São Paulo: Editora Paz eTerra.

HEIMANN, L. S.; CASTRO, I. E. N.; KAYANO, J.; ROCHA, J. L.; RIEDEL, L. F.; TERENCE, M. F.; LUIZ, O. C.; FRAISSAT, R. A.; PESSOTO, U. C. \& JUNQUEIRA, V., 1998. A Descentralização do Sistema de Saúde no Brasil: Uma Proposta de Investi gação sobre o Impacto de Políticas. São Paulo: Instituto de Saúde, Secretaria de Estado da Saúde de São Paulo. (mimeo.)

MONTEIRO, C. A. \& NAZÁRIO, C. L., 1995. Declínio da mortalidade infantil e eqüidade social: O caso da cidade de São Paulo entre 1973 e 1993. In: Ve Ihos e Novos Males da Saúde no Brasil - A Evolução do País e de suas Doenças (C. A. Monteiro, org.), pp. 173-185, São Paulo: Editora Hucitec/ Núcleo de Pesquisas Epidemiológicas em Nutrição e Saúde, Universidade de São Paulo.

MONTEIRO, M. F. G., 1986. Considerações sobre os fatores sócio-econômicos e diferenciais de mortalidade infantil nas regiões metropolitanas do Brasil, calculados através do método caso-controle. In: Perfil Estatístico de Crianças e Mães no Brasil (Fundação Instituto Brasileiro de Geografia e Estatística, org.), pp. 78-92, Rio de Janeiro: Fundo das Nações Unidas para a Infância/ Fundação Instituto Brasileiro de Geografia e Estatística.
NAHAS, M. I. P. \& MARTINS, V. L. A. B., 1995. O índice de qualidade de vida urbana para Belo Horizonte: A elaboração de um novo instrumento de gestão municipal. In: 19o Encontro Anual da Associação Nacional dos Programas de Pós-Graduação em Administração, Anais, no 8, v. 1, pp. 337-350, João Pessoa: Associação Nacional dos Programas de Pós-Graduação em Administração.

NAHAS, M. I. P.; MARTINS, V. L. A.; GUERRA, L. P.; SIMÕES, R. F. \& ESTEVES, O. A., 1997. O índice de qualidade de vida urbana de Belo Horizonte: Um processo de geração de indicadores sociais. Cadernos de Ciências Sociais, 5:88-109.

NAHAS, M. I. P.; RIBEIRO, C. A.; ESTEVES, O. A.; MOSCOVITCH, S. K. \& MARTINS, V. L. A., 1999. O mapa da exclusão social de Belo Horizonte: Metodologia de construção de um instrumento de gestão urbana. Cadernos de Ciências Sociais, 7:7588.

OLIVEIRA, F., 1998. Os Direitos do Antivalor - A Economia Política da Hegemonia Imperfeita. Petrópolis: Editora Vozes.

PAIM, J. S.; COSTA, M. C. N.; CABRAL, V.; M OTA, I. A. \& NEVES, R. B. B., 1987. Spatial distribution of infant mortality and certain socioeconomic variables in Salvador, Bahia, Brazil. Bulletin of the Pan American Health Organization, 21:225-239.

PREFEITURA DE BELO HORIZONTE, 1996. Índice de Qualidade deVida Urbana. Belo Horizonte: Secretaria Municipal de Planejamento.

PREFEITURA DE BELO HORIZONTE, 1998. Memórias do Orçamento Participativo de 1994 a 1997. Belo Horizonte: Secretaria Municipal de Planejamento.

PT (Partido dos Trabalhadores), 1992. Proposta de Governo para uma Bel o Horizonte Democrática e Popular - Síntese dos Programas Setoriais. Belo Horizonte: Diretório Municipal do Partido dos Trabalhadores.

SES-SP (Secretaria de Estado da Saúde de São Paulo), 1998. Critérios para Avaliação e Classificação de Maternidades. São Paulo: SES-SP.

SILVA, L. M. V.; PAIM, J. S. \& COSTA, M. C., 1999. Desigualdades na mortalidade, espaço e estratos sociais. Revista de SaúdePública, 33:187-197.

VICTORA, C. G.; BARROS, F. C. \& VAUGHAN, J. P., 1988. Epidemiologia da Desigualdade- Um Estudo Longitudinal de 6.000 Crianças Brasileiras. São Paulo: Editora Hucitec.

WHITEHEAD, M., 1990. The Concepts and Principles of Equity and Health. Copenhagen: World Health Organization.

Recibido el 19 de marzo de 2001

Versión final presentada el 23 de octubre de 2001

Aprobado el 14 de noviembre de 2001 